\title{
q-deformed dynamics and Josephson junction
}

\author{
Ramandeep S. Johal* \\ Department of Physics, Panjab University, \\ Chandigarh-160 014, India.
}

\begin{abstract}
We define a generalized rate equation for an observable in quantum mechanics, that involves a parameter $q$ and whose limit $q \rightarrow 1$ gives the standard Heisenberg equation. The generalized rate equation is used to study dynamics of current biased Josephson junction. It is observed that this toy model incorporates diffraction like effects in the critical current. Physical interpretation for $q$ is provided which is also shown to be $q$ deformation parameter.
\end{abstract}

*e-mail: rrai@lycos.com 
q-deformed algebras [1], 2] and their various realizations have been subject of intense study in the past years. They provide a platform to study alternative schemes of quantization [3] whereby issues like generalized statistics [4] can be discussed. The effect of deformation has been studied also for different quantum mechanical toy models [5, 6] and these algebras are finding applications as dynamical symmetry algebras of various physical systems. Roughly speaking, $q$-deformed version of a theory corresponds to its formulation on a lattice and $q$ parameter may be interpreted as equivalent to lattice spacing [7]. Appropriately, it is also interesting to observe that some theories can be viewed as intrinsically $q$-deformed, and thus use of $q$-deformed algebras appears naturally in certain contexts, like Barnett-Pegg theory of rotation angle [8], nonextensive entropy within generalized statistical mechanics [9], Bloch electron problem [10] and so on.

Apart from the $q$-deformation of the algebraic structure for model physical systems, attempts have been made to $q$-generalize Schrödinger equation [11], Dirac equation [12 as well as Heisenberg equation of motion [13]. In this paper, we focus on the deformation of Heisenberg equation, which gives the rate of change of an observable $\hat{A}$ as

$$
\dot{\hat{A}} \equiv \frac{d \hat{A}}{d t}=\frac{1}{i \hbar}[\hat{A}, \hat{H}]
$$

Now a simple $q$-deformation of this equation, would be to make the rate of change proportional to modified commutator $[\hat{A}, \hat{H}]_{q}=\hat{A} \hat{H}-q \hat{H} \hat{A}$. However, in this approach the hamiltonian is no longer constant of motion, as pointed 
out in [13].

Our purpose here is to propose a generalized rate equation for an observable in quantum mechanics. We apply it to study the dynamics of Josephson tunnel junction (JJ) [14. The new definition involves a parameter which will be interpreted as $q$-deformation parameter and it will be discussed that it does lead to a physical effect.

We first present a brief summary of the standard way of writing Heisenberg equation of motion for a JJ. Following [15], the time dependent hamiltonian of a current biased JJ is given by

$$
\hat{H}=\frac{1}{2 C}\{2 e \hat{n}+I t\}^{2}-E_{J} \cos \hat{\phi}
$$

$C, e, I$ respectively are the junction capacitance, electronic charge, current through the junction and $E_{J}$ is the Josephson coupling energy between two superconductors across the junction. $\hat{n}$ measures the number of cooper pairs transferred across the junction and $\hat{\phi}$ is the phase operator canonically conjugate to $\hat{n}$, in the sense that $[\hat{n}, \hat{\phi}]=-i$, which may be satisfied by the differential realization, $\hat{n}=-i \frac{\partial}{\partial \phi}$. It may be remarked that $\hat{n}$ and $\hat{\phi}$ are hermitian operators and $\cos \hat{\phi}$ may be written in terms of unitary exponential operators as $\cos \hat{\phi}=\left(e^{i \hat{\phi}}+e^{-i \hat{\phi}}\right) / 2$.

Now applying Eq. (11) for the case of $\hat{\phi}$ and $\hat{n}$ (and using alongwith $\left.\left[\hat{n}, e^{i \hat{\phi}}\right]=e^{i \hat{\phi}}\right)$, we get

$$
2 e \dot{\hat{n}}=-I_{J} \sin \hat{\phi}, \quad(\hbar C / 2 e) \dot{\hat{\phi}}=2 e \hat{n}+I t,
$$


where $I_{J}=2 e E_{J} / \hbar$ is the critical value of current, beyond which a finite voltage appears across the junction.

Combining the two equations in (3), we write

$$
(\hbar C / 2 e) \ddot{\hat{\phi}}+I_{J} \sin \hat{\phi}=I .
$$

In case, $E_{J}$ is quite larger than the electrostatic charging energy $E_{C}=2 e^{2} / C$ (due to transfer of one cooper pair across the junction), we can replace the operators by their expectation values and so the "classical" equation of motion corresponding to Eq. (4) is

$$
(\hbar C / 2 e) \ddot{\phi}+I_{J} \sin \phi=I
$$

which implies motion of a classical particle in a washboard potential

$$
U(\phi)=-\frac{2}{\hbar e}\left(I_{J} \cos \phi+I \phi\right)
$$

In the absence of fluctuations, $\dot{\phi}=0$, and we have a minimum in potential energy, $\frac{\partial U}{\partial \phi}=0$ obtaining

$$
I=I_{J} \sin \phi
$$

In the following, we generalize the definition of rate of change of an operator with respect to time. Let us illustrate for an arbitrary observable $\hat{A}$. Define

$$
\mathcal{D}_{t} \hat{A}=\frac{q^{-\hat{A}}}{\ln q} \frac{d q^{\hat{A}}}{d t} .
$$

The operator defined above is not equal to $d \hat{A} / d t$ if $\hat{A}$ and $d \hat{A} / d t$ do not commute. This can be seen by expanding $q^{\hat{A}}$ as power series. However, as 
$q \rightarrow 1$, the above operator approaches $d \hat{A} / d t$. Also $d q^{\hat{A}} / d t$ is given by the standard Heisenberg equation of motion

$$
\frac{d q^{\hat{A}}}{d t}=\frac{1}{i \hbar}\left[q^{\hat{A}}, \hat{H}\right] .
$$

Thus we obtain

$$
\mathcal{D}_{t} \hat{A}=\frac{q^{-\hat{A}}}{i \hbar \ln q}\left[q^{\hat{A}}, \hat{H}\right] .
$$

So as $q \rightarrow 1$, the right hand side approaches $[\hat{A}, \hat{H}] / i \hbar$ and in this sense, Eq. (10) can be considered as $q$-generalization of the standard Heisenberg equation. We later on identify the parameter $q$ as the one appearing in $q$-deformation theory.

To apply the new rate equation to Josephson junction, we alternately consider $\hat{A}$ to be operator $\hat{\phi}$ and $\hat{n}$. Also from now on we take $q=\exp (i s)$ with $s$ real. As a consequence, we obtain

$$
(\hbar C / 2 e) \mathcal{D}_{t} \hat{\phi}=2 e \hat{n}+I t+e s
$$

and

$$
2 e \mathcal{D}_{t} \hat{n}=-I_{J} \frac{\sin (s / 2)}{s / 2} \sin (\hat{\phi}-s / 2) .
$$

Compare these two equations with the original Heisenberg equations, Eq. (3). Naturally, the above Eqs. reduce to the latter, as $s \rightarrow 0($ or $q \rightarrow 1$ ). Following the similar arguments (as after Eq. (3)), we can identify the critical current of the modified problem as following

$$
I=I_{J} \frac{\sin (s / 2)}{s / 2} \sin (\phi-s / 2) .
$$


There are two consequences of $s$ being not equal to zero; 1 ) there is phase shift, that in standard JJ may be produced by, for example, external magnetic field. It is this phase shift that accounts for interference in SQUID devices, where more than one JJs are coupled, 2) more remarkably, we see that the maximum critical current $I_{J}$ is modified by a Fraunhoffer like diffraction term. This pattern is observed in junctions with rectangular geometry in the presence of applied magnetic field [16]. This is a consequence of the finite width of the junction and is analogous to the effect in optics. Comparing with the standard result, we have $s=2 \pi \Phi / \phi_{0}$, where $\Phi$ is the effective flux linked with the junction and $\phi_{0}=\pi \hbar / e$.

Next, we would like to interpret parameter $q$ as the one occuring in $q$ analysis. Note that using the realization $\hat{n}=-i \partial / \partial \phi$, we can show

$$
q^{\hat{n}} e^{i \hat{\phi}}=q e^{i \hat{\phi}} q^{\hat{n}}
$$

Now in the theory of $q$-calculus [7], the relation $\hat{X} \hat{Y}=q \hat{Y} \hat{X}$ plays important role. Working in the eigenspace of $\hat{X}$, we have $\hat{X}|n\rangle=x|n\rangle$, where $n$ labels the eigenstates. On this space, $\hat{Y}$ acts like a shift operator, $\hat{Y}|n\rangle=|n+1\rangle$. For the present case, we identify $X=q^{\hat{n}}$ and $|n\rangle$ as the pair-number difference states. Here $n=0, \pm 1, \pm 2, \cdots$. Note that $n$ counts the number of cooper pairs transferred across the junction and the sign \pm refers to the direction (positive for transfer in one direction and negative for opposite direction). Also $|n\rangle$ is the appropriate basis in which the present hamiltonian Eq. (2), describes tunnelling of cooper pairs. Now the dual basis to the above is given 
by

$$
|\phi\rangle=(2 \pi)^{-1 / 2} \sum_{n=-\infty}^{\infty} e^{-i n \phi}|n\rangle,
$$

and the eigenvalue equation, $e^{i \hat{\phi}}|\phi\rangle=e^{i \phi}|\phi\rangle$, implies $e^{i \hat{\phi}}|n\rangle=|n+1\rangle$. Thus it is natural to identify $\hat{Y}=e^{i \hat{\phi}}$, satisfying Eq. (14). Also, $e^{-i \hat{\phi}}$ transfers the cooper pair in the opposite direction. It may be remarked that incorporating negative eigenvalues of $\hat{n}$ is important to maintain unitarity of the shift operator $e^{i \hat{\phi}}$, and hence the hermiticity of operator $\hat{\phi}$.

In the above, we used the standard JJ hamiltonian with a generalized definition of rate of change of an observable and arrived at generalized rate equations for the JJ. It may be asked whether same generalized dynamics can be obtained by using a modified or deformed hamiltonian in the standard Heisenberg equation. For the JJ case, it is easy to give such a hamiltonian as follows

$$
\hat{H}_{s}=\frac{1}{2 C}\{2 e(\hat{n}+s / 2)+I t\}^{2}-E_{J}^{\prime} \cos (\hat{\phi}-s / 2),
$$

where now the coupling energy becomes $s$ dependent as

$$
E_{J}^{\prime}=E_{J} \frac{\sin (s / 2)}{s / 2} .
$$

We note that $\hat{H}_{s}$ involves operators $\hat{n}$ and $\hat{\phi}$ both shifted by $s / 2$ (with opposite signs). To interpret the parameter $s$ in the modified hamiltonian, we consider the hamiltonian for a charged particle moving on a ring in the xy-plane

$$
\hat{H}=\frac{\hat{L}_{z}^{2}}{2 M}-V_{0} \cos \hat{\phi}
$$


where $M$ is the moment of inertia. Angular momentum operator $\hat{L}_{z}$ is the analogue of operator $\hat{n}$ in the case of JJ above and satisfies $\left[\hat{L}_{z}, \hat{\phi}\right]=i \hbar$. Now the standard equation of motion for $\hat{\phi}$ is given by $\dot{\hat{\phi}}=\hat{L}_{z} / M$. The generalized rate of change comes out to be

$$
\mathcal{D}_{t} \hat{\phi}=\left(2 \hat{L}_{z}+s\right) / 2 M
$$

It is clear that the following hamiltonian

$$
\hat{H}_{s}=\frac{\left(\hat{L}_{z}+s / 2\right)^{2}}{2 M}-V_{0} \cos \hat{\phi}
$$

when employed in the standard rate of change, gives the generalized dynamics. Now the deformed hamiltonian Eq. (20) is just the one for a charged particle moving on a ring at whose center is magnetic flux proportional to $s$. Thus once again, we get the same interpretation of parameter $s$ as representing a fictitious flux.

Concluding, we have considered a generalized rate equation for an observable in quantum mechanics, which goes over to standard Heisenberg equation as the parameter $q=\exp (i s)$ goes to unity. To study its implications, we have applied it to current biased Josephson tunnel junction. We saw that this toy model can incorporate the diffraction like effects of critical current for rectangular JJs. This allows to interpret parameter $s$ as equivalent to magnetic flux through the junction. Also we were able to represent $q$ as deformation parameter occuring in the theory of $q$-analysis. Finally, we considered deformation of the system hamiltonian and again could infer that $s$ 
represents a magnetic flux threading the Josephson junction or the analogue system of charged particle on a ring.

\section{Acknowledgement}

The author gratefully acknowledges Prof. K.N. Pathak for discussion and encouragement. This work was supported by Department of Science and technology, India.

\section{References}

[1] V.G. Drinfeld, Sov. Math. Dokl. 32 (1985) 254; L. Faddeev, N. Reshetikhin and L. Takhtajan, Algebra and Analysis. 1 (1989) 178; S.L. Woronowicz, Publications RIMS, Kyoto Univ. 23 (1987) 117.

[2] M. Jimbo, Lett. Math. Phys. 10 (1985) 63.

[3] K. Odaka, T. Kishi and S. Kamefuchi, J. Phys. A 24 (1991) L711; D. Bonatsos and C. Daskaloyannis, Phys. Lett. B 307 (1993) 100.

[4] O.W. Greenberg, U. Maryland preprint 93-097 (1993).

[5] J. Schwenk and J. Wess, Phys. Lett. B 291 (1992) 273.

[6] E.G. Floratos, in: Proc. Argonne Workshop on Quantum Groups, eds. T. Curtright, D. Fairlie and C. Zachos (World Scientific, Singapore, 1991) p. 158; T. Kobayashi and T. Suzuki, Phys. Lett. B 317 (1993) 359. 
[7] A. Dimakis and F. Müller-Hoissen, Phys. Lett. B 295 (1992) 242.

[8] S. Abe, Phys. Rev. A 54 (1996) 93.

[9] R.S. Johal, Phys. Rev. A 58 (1998) 4147.

[10] P.B. Weigmann and A.V. Zabrodin, Phys. Rev. Lett. 72 (1994) 1890; Y. Hatsugai, M. Kohmoto and Y.S. Wu, Phys. Rev. Lett. 73 (1994) 1134 .

[11] S. Chaturvedi, R. Jagannathan, R. Sridhar and V. Srinivasan, J. Phys. A 26 (1993) L105.

[12] J. Lukierski, A. Nowicki and H. Ruegg, Phys. Lett. B 293 (1992) 344; A. Nowicki, E. Sorace and M. Tarlini, Phys. Lett. B 302 (1993) 419.

[13] M. Chaichian and D. Ellinas, J. Phys. A 23 (1990) L291.

[14] B.D. Josephson, Phys. Letters 1 (1962) 251; Rev. Mod. Phys. 36 (1964) 216.

[15] E. B-Jacob and Y. Gefen, Phys. Lett. A 108 (1985) 289.

[16] R.C. Jaklevic, J. Lambe, J.E. Mercereau and A.H. Silver, Phys. Rev. A 140 (1965) 1628. 